\title{
Guest Editorial \\ Special Issue on Selected Papers From the IEEE Sensors Conference 2018
}

$\mathbf{S}$ INCE its inception in 2002, the IEEE Sensors Conference has come a long way in establishing itself as a premier conference in the world on sensors and sensing technologies. With the entire world moving rapidly toward a ubiquitous intelligence society, the emphasis on deployment of trillions of sensors in the near future is driving the world of sensor technology like never before. It is reflected in the rapidly increasing research and development activities on sensors throughout the world today. The IEEE Sensors Council has done a remarkable job in promoting this area and providing a consistent platform for sharing and dissemination of knowledge related to all aspects of sensing and sensor development among researchers, manufacturers, and the users of sensors. The idea of holding the IEEE Sensors Conference in different parts of the world actively supports this promotion over different geographies. It could not have been more timely to hold SENSORS 2018, for the first time, in one of the largest and fastest developing economies of the world - India. The 2018 edition of the Sensors Conference was held on October 28-31 in New Delhi - the capital city of India that has been inhabited for six thousand years and has seen a great many upheavals in history.

The call for papers for SENSORS 2018 attracted 921 paper submissions. As with every edition of the conference, these papers went through a rigorous peer review process, with each paper reviewed by at least three reviewers. The list of reviewers included not just the Track Chairs but also 589 reviewers who, on average, reviewed at least five papers each. After reviews, all papers went through a detailed scrutiny by the Technical Program Committee (TPC) that comprised the TPC Co-Chairs - Prof. Rudra Pratap and Prof. Ravinder Dahiya, along with 38 Co-Chairs of 19 Technical Tracks. At the end, 485 papers were accepted based on their originality and quality of work, with 234 papers selected for oral presentation and 251 for poster presentation. These papers represented work done by researchers from 58 countries. The most impressive and satisfying statistics was that a whopping 643 papers (70\% of the total) were submitted from the Asia Pacific region. Thus the objective of taking the conference to different parts of the world for encouraging more participation from the local region was more than achieved. At the end,

Digital Object Identifier 10.1109/JSEN.2020.2984165 the Sensors Council's commitment to promote research and development on sensors across the world got a big boost from such enthusiastic participation from the Asia Pacific region.

The papers presented at SENSORS 2018 were spread over the regular 12 Tracks of the conference, another Track for Industrial Practice, a special Track for Live Demonstrations, and four Focused Tracks covering Remote Sensing and Satellite Navigation, Wearable and Implantable Biomedical Sensors, Inertial Sensor Systems, and Sensor Systems for Neurological Assessment and Clinical Studies. In addition, a special track was provided to encourage presentation of some papers already published in IEEE SENSORS LETTERS and IEEE SENSORS JOURNAL that came out after the 2017 edition of the conference and the authors did not get an opportunity to present those papers at this forum. All presented papers, except those comprising this special track, were published on IEEE Xplore.

Of all regular accepted contributions, the authors of the top 30 papers by originality score were invited to submit an extended version of their papers, if they wished to be included in a special issue of the IEEE SENSORS JOURNAL dedicated to the Sensors Conference. The authors were informed that their submissions would go through the same rigorous review process that the regular submissions to IEEE SENSORS JOURNAL do. This review process was handled by the Guest Editors of this Special Issue. Finally, from among those papers that were submitted in response to our invitations, we have accepted 12 papers for the Special Issue that you are reading right now.

First we would like to thank the authors who chose to accept our invitation and submit extended versions of their papers. We also thank all the reviewers who reviewed these papers for the Special Issue and provided detailed comments. Most of these papers went through a revision in response to the reviewers' questions and comments. We were very particular about accepting papers only after the peer review process was complete in all its rigor. Thus, these papers have come to this Special Issue through a rather long process starting with the conference paper review process that selected them first for presentation at SENSORS 2018. We would like to express our gratitude to all those reviewers for their precious time.

The Guest Editors would like to thank Ms. Lauren Young and Ms. Leigh Ann Testa for their support in the publication of 
this issue. We also express our gratitude to the Editor-in-Chief of IEEE SEnsors Journal, Prof. Sandro Carrara, for his encouragement and continuous support for a successful publication of this Special Issue.

The Guest Editors (in alphabetical order by last name).

RAVINDER DAHIYA, Guest Editor

James Watt School of Engineering

University of Glasgow

Glasgow G12 8QQ, U.K.

e-mail: ravinder.dahiya@glasgow.ac.uk
OMer Oralkan, Guest Editor

Electrical and Computer Engineering

North Carolina State University

Raleigh, NC 27695 USA

e-mail: ooralka@ncsu.edu

RUDRA PRATAP, Guest Editor

Centre for Nano Science and Engineering

Indian Institute of Science

Bengaluru 560012, India

e-mail: pratap@iisc.ac.in 